\title{
Spatiotemporal integration of molecular and anatomical data in virtual reality using semantic mapping
}

\author{
Jung Soh' \\ Andrei L Turinsky' \\ Quang M Trinh' \\ Jasmine Chang' \\ Ajay Sabhaney' \\ Xiaoli Dong' \\ Paul MK Gordon' \\ Ryan PW Janzen' \\ David Hau ${ }^{2}$ \\ Jianguo $\mathrm{Xia}^{2}$ \\ David S Wishart ${ }^{2}$ \\ Christoph W Sensen' \\ 'Sun Center of Excellence for Visual \\ Genomics, University of Calgary, \\ Calgary, AB, Canada; ${ }^{2}$ Department \\ of Biological Sciences, University \\ of Alberta, Edmonton, AB, Canada
}

Correspondence: Christoph W Sensen Sun Center of Excellence for Visual Genomics, HS I I50, Faculty of Medicine, 3330 Hospital Drive NW, Calgary, AB T2N 4NI, Canada

$\mathrm{Tel}+\mathrm{I} 403220430$ I

$\mathrm{Fax}+\mathrm{I} 4032109399$

Email csensen@ucalgary.ca

\begin{abstract}
We have developed a computational framework for spatiotemporal integration of molecular and anatomical datasets in a virtual reality environment. Using two case studies involving gene expression data and pharmacokinetic data, respectively, we demonstrate how existing knowledge bases for molecular data can be semantically mapped onto a standardized anatomical context of human body. Our data mapping methodology uses ontological representations of heterogeneous biomedical datasets and an ontology reasoner to create complex semantic descriptions of biomedical processes. This framework provides a means to systematically combine an increasing amount of biomedical imaging and numerical data into spatiotemporally coherent graphical representations. Our work enables medical researchers with different expertise to simulate complex phenomena visually and to develop insights through the use of shared data, thus paving the way for pathological inference, developmental pattern discovery and biomedical hypothesis testing.
\end{abstract}

Keywords: anatomical atlas, gene expression, pharmacokinetics, biomedical data integration, CAVEman, virtual reality in medicine

\section{Introduction}

Biomedical data produced while studying an organism using a number of lab techniques often exist in a wide variety of data formats, which are often incompatible with each other. The spatial granularity of such data can vary greatly, from the molecular level (eg, models of elucidated molecular complexes) to the macroscopic level (eg, volumetric scans of tissues and organs). ${ }^{1}$ Existing integration techniques for multi-database systems, data warehousing and federated database systems ${ }^{2}$ are often inadequate for handling the abundance of biomedical data, because of the diversity, complexity, and the ever-changing nature of the biomedical data sources. Most biomedical data sources, such as genome sequences, proteomics data, metabolomic information, and anatomical data, are created and managed independently, presenting a number of challenges when data from multiple sources need to be integrated in order to create a solution to complex biomedical queries.

We have created an integrated ontology-based framework that enables semantic interoperability between multiple autonomous biomedical data sources with different semantics. Using two case studies, one involving gene expression data and the other using pharmacokinetic data, we describe how the framework combines a set of molecular data with an anatomical atlas of 3D human body. Our source of anatomical data is the CAVEman project (http://www.4dbioinformatics.ca), which provides a standardized anatomical context for the exploration of biomedical datasets in a virtual reality environment using an object-oriented model of the human body. The anatomical component of CAVEman ${ }^{3}$ is based on the $3 \mathrm{D}$ digital atlas of the human 
adult male anatomy developed by Kasterstener Inc, expanded from its earlier version, ${ }^{4}$ which is structured according to the nomenclature defined in Terminologia Anatomica. ${ }^{5,6}$

\section{Integration framework}

The goal of our integration framework is to enable the user to visualize an integrated representation of biomedical data coming from separate data sources (Figure 1). The platform for this integration is the human body atlas of the CAVEman system. The user queries a biomedical data source for a subset of data to be visualized. The data query interface formats the user query depending on the type of the data to retrieve the desired instances of data. The data is then partitioned into anatomical information (eg, body systems or organs) and symbolic information (eg, numbers or positive/negative indicators), which are separately mapped for subsequent integration. The semantic mapper finds the mapping from the components of anatomical information to standardized terms or IDs adopted in the human body atlas. This process generates a set of unique indices for the relevant three-dimensional (3D) graphic models of the anatomical objects. The visual mapper produces a graphic representation of the symbolic information to be viewed, using selected visual features such as color, shape, and texture. Symbolic information is converted to a set of visually salient features that also represents the original information unambiguously. In a virtual reality environment, renderings of the visual features on the selected models of the human body atlas are generated and presented to the user.

Semantic mapping is required because there are a wide variety of different anatomical terminologies used in biomedical data sources. ${ }^{7}$ Nonstandard terms referring to organs and tissues need to be mapped to standardized identifiers for the anatomical parts of interest. The actual methods of finding the semantic mapping vary according to the type of data. Visual mapping is employed to convert symbolic (ie, textual and numerical) information to visual features that can be represented unambiguously in a virtual reality environment. The selection of the specific visual features to be used is guided by the ranges of values to represent and the methods used for normalizing them. We achieve the integration in 3D space by combining semantic mapping and visual mapping. If the data to be visualized is time-dependent, spatiotemporal mapping is obtained by repeatedly applying this spatial mapping framework over the existing time points. We show the applicability of this generic framework to integrating different types of data sources with the human body anatomical atlas by using two case studies.

\section{Case study I: Gene expression data mapping}

The problem

Our first case study is on integration of genomic and anatomical data, which involves visual mapping of gene expression

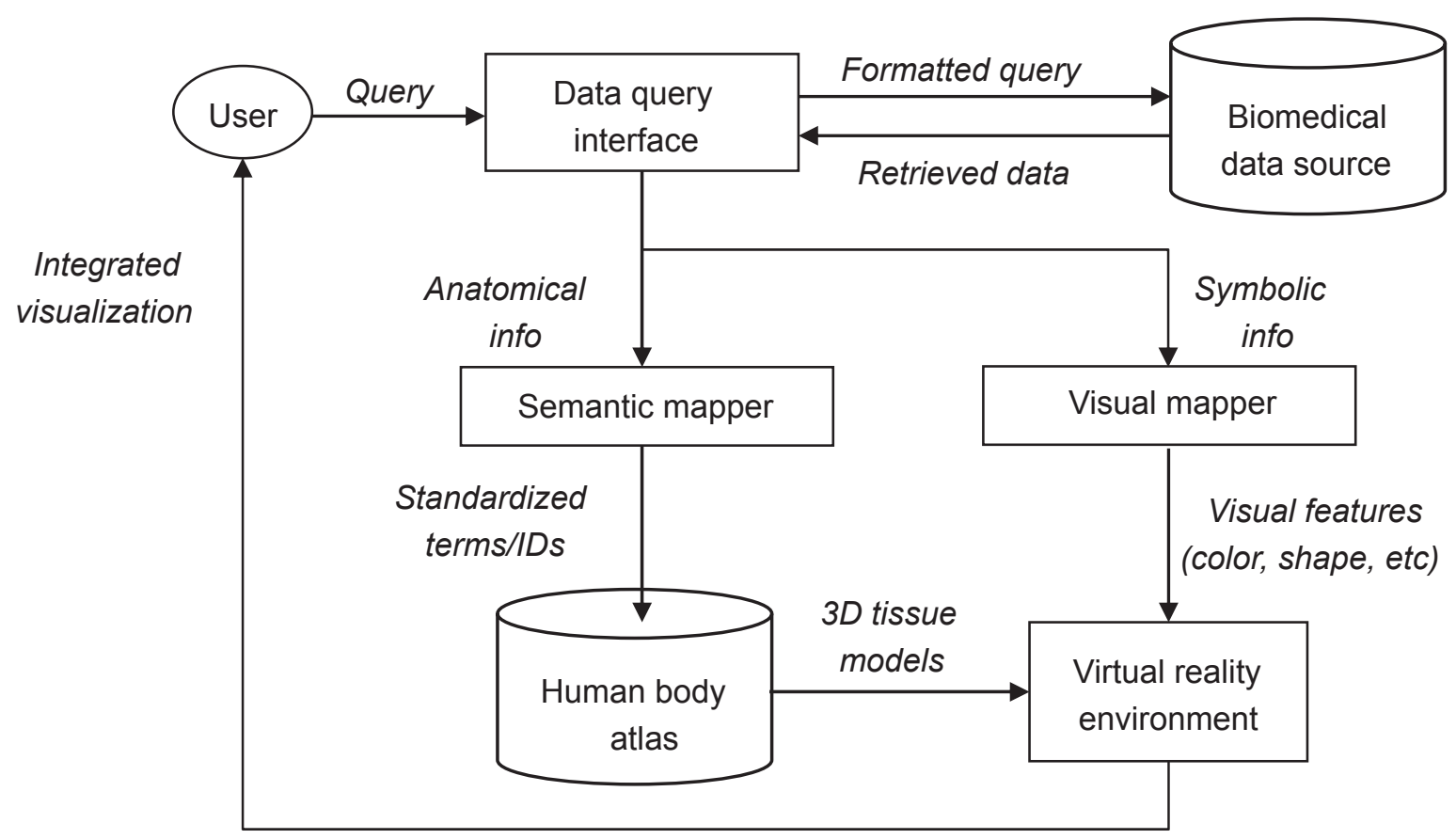

Figure I A computational framework for spatial integration of molecular and anatomical data. The anatomical information contained in the molecular data is semantically mapped to indices to a human body atlas. The textual or numerical information is mapped to visual features to be rendered on the retrieved 3D models of the human body in a virtual reality environment. 
data onto the anatomical context provided by the human body atlas. The amount of gene expression information generated from microarray experiments has been growing at an exponential rate. ${ }^{8}$ As those experiments employ high-throughput methods, a tremendous amount of data is created even from a single experiment. While statistical analysis of gene expression data can reveal interesting patterns, presenting the analysis results in a visually intuitive way should also be an essential part of any gene expression analysis program. The visual aspect of dataset presentation is a significant factor that provides intuitive insights into patterns and leads to conclusions, which might be missed when using only statistical assessments.

Most software for gene expression data analysis performs unsupervised clustering to generate meaningful groups of genes without bias of prior knowledge of gene function. However, the high dimensionality of the datasets often hinders the discovery of interesting patterns, clusters, and outliers, making it difficult to classify genes into meaningful groups automatically. Researchers often have to refer to many kinds of specific genomic and anatomical information while maintaining an overall view of the gene expression dataset, in order to make sense of the analysis results even if they are visually presented (eg, heat maps). Visual mapping of gene expression values onto a highly relevant context, such as the tissues themselves from which mRNA expression values were experimentally measured, would provide the user a coherent view of the vast array of the expression values. To this end, a system for gene expression profiling should be able to integrate as much biologically and statistically relevant information as possible and provide the user with a coherent simultaneous view of such pieces of information.

\section{The data}

The gene expression data has been retrieved from Genomics Institute of the Novartis Research Foundation (GNF) SymAtlas, ${ }^{9}$ a publicly available database of gene function and structure (http://symatlas.gnf.org). In particular, we use the Human U133A/GNF1H microarray dataset, which has been pre-normalized using the GC content adjusted Robust Multi-array Average (gcRMA) algorithm. ${ }^{10}$ The dataset contains gene expression values for 44,760 probe sets in 79 human organs and tissues, obtained using Affymetrix probe set identifiers. ${ }^{11}$

\section{Methods}

We applied the integration framework of Figure 1 to integrate gene expression data with anatomical data. The implementations of the semantic mapper and visual mapper are dictated by the characteristics of the gene expression data we wanted to visualize. Figure 2 illustrates the methods and the data flow for gene expression data mapping, which is based on the integration framework.

The semantic mapper implementation is based on ontologies, which formalize vocabularies defined by data owners, their properties, and their semantic relationships and restrictions within a knowledge domain. ${ }^{12,13}$ We use the Web Ontology Language (OWL; http://www.w3.org/2004/owl) to establish semantic relationships among entities from separate biomedical datasets. For each data source, an OWL ontology generator creates a local ontology that describes the data source, and translates the data into instances of the newly created ontology. The data owners then define the relationships between the elements of the newly generated ontology and the semantic constructs from other related ontologies.

For our case study, we use two local ontologies in the form of OWL resources. The first one represents the GNF SymAtlas dataset that lists the available genes, the tissues in which their expression were measured, and the corresponding microarray spot intensity values (SymAtlas ontology). This ontology contains 44,760 terms describing the genes, where each gene term contains 79 terms describing spot intensity values from different organs and tissues. The second one describes the tissue names used in GNF SymAtlas and their mapping to the standard Terminologia Anatomica terms (tissues ontology). This ontology contains 79 terms, as it only describes the mapping from the anatomical terms for the organs and tissues used in the dataset to proper TA IDs. The mapping is sometimes not straightforward, as the SymAtlas anatomical terms are not always directly mappable to a TA term. Thus, the actual mapping was done not by the providers of the SymAtlas data, but by the researchers for this case study. We have modified the Pellet reasoning engine ${ }^{14}$ to validate semantic relationships between ontologies automatically. The mapping from a gene ID to tissue names to TA IDs is automatic due to the use of this ontology reasoner.

The semantic mapper starts with a search for the instances the Affymetrix ID of a queried gene in the SymAtlas ontology. Genes are referred to by the corresponding Affymetrix probe set identifiers: for example, 1007_s_at is the Affymetrix probe set ID of a human epithelial discoidin domain-containing receptor 1 precursor gene. The data available for this gene is contained within the affymetrix:gene_1007_s_at element of the SymAtlas ontology file. Once matching records are found in the OWL-formatted data, the associated information, 


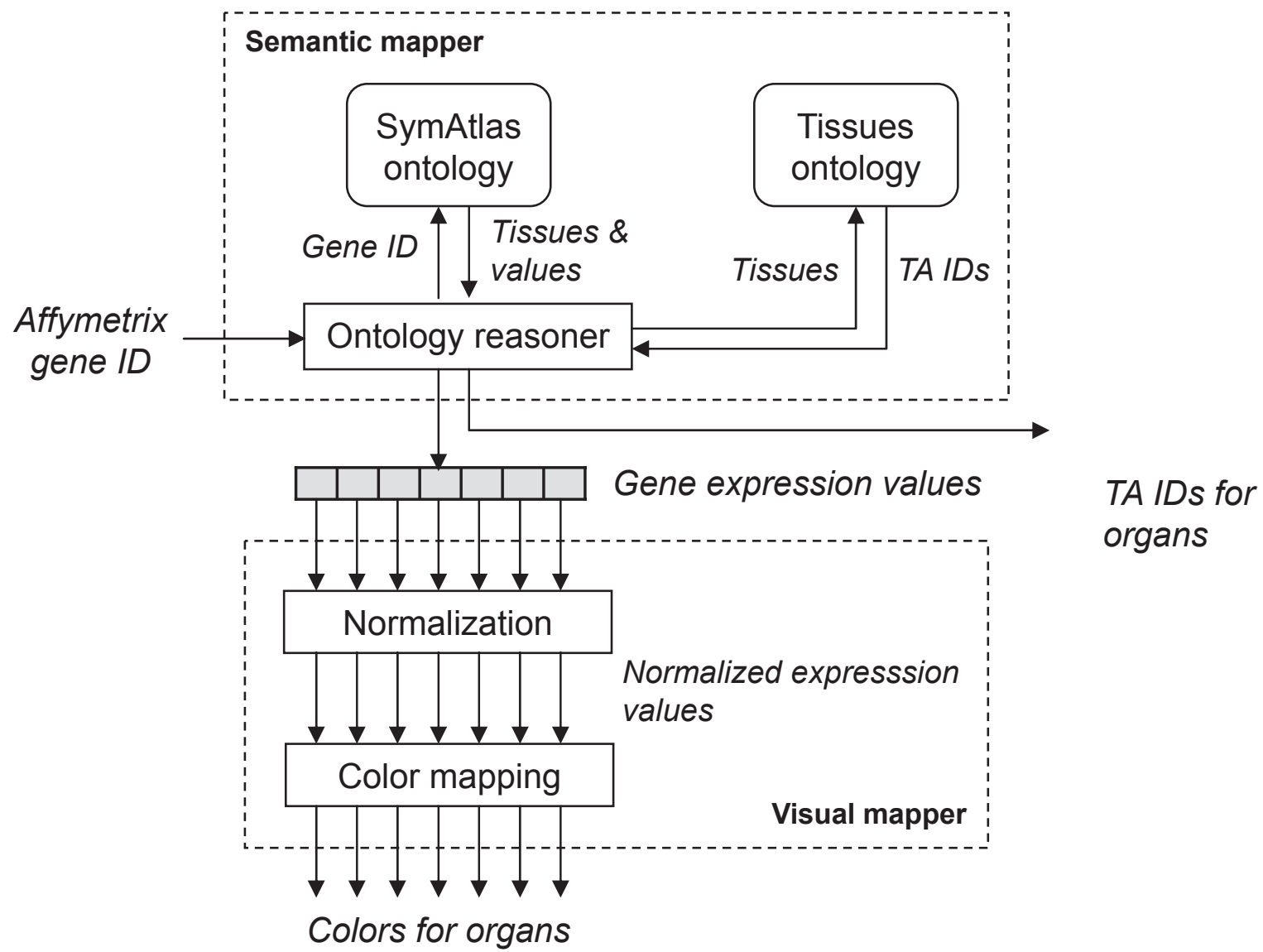

Figure 2 Semantic mapping and visual mapping for integrated visualization of gene expression data for a queried gene. The semantic mapper maps anatomical terms to organ IDs in TA using the ontologies. The visual mapper normalizes gene expression values with respect to a control organ and converts them into color values to be used for rendering the organs.

including terms that describe the cell line or tissue in which the expression was measured and the spot intensities of such measurements as recorded in the SymAtlas data source, is retrieved. The tissue ontology is used for relating tissue names used in the SymAtlas data to the corresponding TA identifiers. For example, "Heart" in SymAtlas data is mapped to the TA ID of "A12.1.00.001."

The role of the visual mapper is to convert the retrieved numerical gene expression values into visual features that can be readily rendered on the human body atlas. We chose to use color as the key visual feature because it can represent a vast range of expression values. The gene expression values are first normalized with respect to a control or reference organ that can be selected by the user. In the case study, any one of the 79 organs can be chosen as the control organ. Thus an expression value that is greater than that for the control organ would be regarded as over-expressed. Using a neutral color for the control organ (eg, white) the expression values for all the other organs are color-mapped to show the strength of the gene expression in the corresponding organs, relative to the control organ. This provision is useful comparing the relative expression levels of a gene in many different organs simultaneously.

\section{Results}

The visualization result for the Affymetrix ID 1007_s_at is shown in Figure 3. It clearly shows very high expression of the gene in the amygdala and the colorectal adenocarcinoma cell lines (mapped onto the 3D rectum model); relatively high expression in olfactory bulbs, the pituitary gland and the trachea; relatively low expression in cerebral peduncles, kidneys, pancreas, pons and tongue tissues; and low expression in the heart and liver tissues. In contrast to the original numerical dataset, which contains a rather overwhelming amount of data (79 spot intensity values in different tissues for each probe set), our 3D visualization gives the user an immediate, easy-to-comprehend visual summary of the highlights of the body-wide expression patterns. With this type of visualization, there is no need for the user to mentally link a multitude of expression values to a collection of tissues in which each value was measured from. The measuring context and the magnitude of the expression values are clearly 


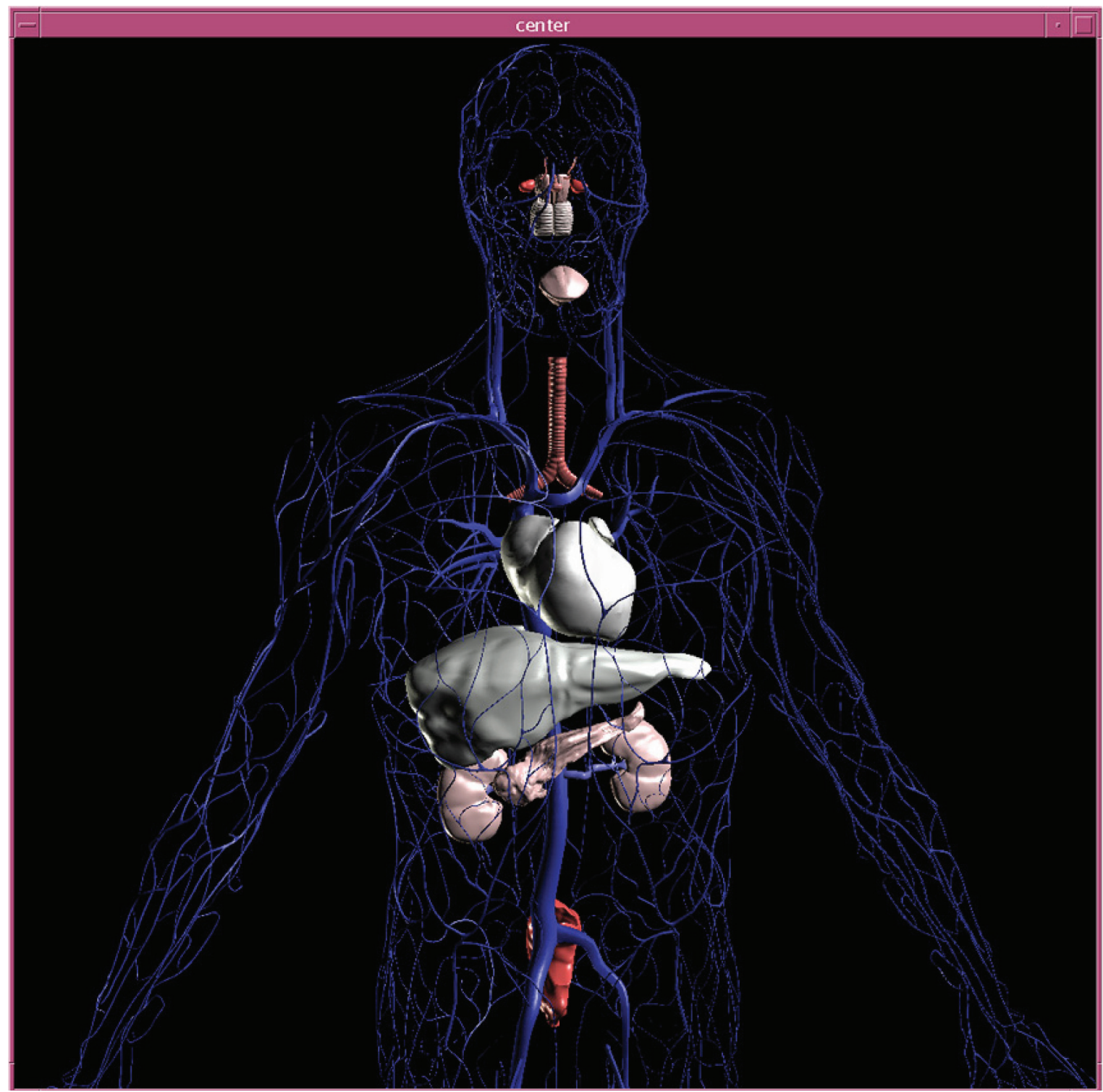

Figure 3 3D visualization of body-wide gene expression patterns for the Affymetrix probe ID 1007_s_at, shown for 12 selected organs. Darker shades of red represent higher expression values.

represented as the anatomical organs displayed and their color, respectively. This helps to alert the human explorer visually to the presence of "interesting" patterns in the data, which can be explored further using the original dataset.

\section{Case study 2: Pharmacokinetic data integration}

\section{The problem}

Our second case study involves a knowledge base provided by the Human Metabolome project, ${ }^{15}$ in particular its DrugBank component. ${ }^{16}$ The goal of the case study is to visually model the absorption, distribution, metabolism, and excretion (ADME) of drugs within the anatomical context. Almost every FDA approved drug must go through rigorous pharmacokinetic analysis, wherein the drug's ADME is determined through a combination of animal experiments and computer modeling. Therefore, creating a mechanism for accurate ADME models and simulations has the potential to contribute to the drug discovery and design processes.

One of the difficulties in pharmaceutical metabolomics is that there is a significant disconnect between the experimental data (which is often visually represented) and the pharmacokinetic models (which are nonvisual). In particular, pharmacokinetic modeling is largely done using simplified 
compartmental models (boxes with different partition coefficients), based on classical differential equations with simplified boundary conditions. This approach may appeal to mathematicians but not necessarily to biologists or medical doctors who are involved in drug development and testing. Likewise, classical methods mostly ignore the importance of visualization as an aid to understanding pharmacokinetic or metabolic processes. Spatiotemporal mapping of pharmacokinetic events onto their proper anatomical context will allow drug developers to: (i) share their results with a broader audience; (ii) assist in teaching pharmacokinetic principles to students and clinicians; (iii) permit real-time exploration and testing of drug ADME; and (iv) better integrate experimental data with theoretical calculations.

\section{The data}

The data was generated using a Java program for ADME modeling, called ADME-Modeler, developed in the Human Metabolome project (http://metabolomics.ca). The software interactively requests the following information from the user: (i) the chemical compounds involved in the ADME reactions; (ii) route of administration (enteral/oral or parenteral/intravenous), volume of distribution, and bioavailability; (iii) the rate constants of absorption, distribution, conversion and elimination for each compound; and (iv) additional information such as time intervals and dosage regimen. The program then calculates the temporal distribution, concentration and location of each of the drugs and drug metabolites using either the first-order elimination or the Michaelis-Menten kinetics ${ }^{17}$ as selected by the user. To calculate compound concentrations in different organs, the human body is conceptually divided into three compartments: (i) the gastric-intestinal compartment including the stomach and the intestine; (ii) the central compartment including highly perfused organs such as the cardiovascular system, liver, kidney, brain, spleen, and lungs; and (iii) the peripheral compartment including cerebrospinal fluid, bone, muscle, and adipose tissue.

ADME and pharmacokinetic information on several drugs were obtained: acetaminophen (Tylenol), acetylsalicylic acid (Aspirin), atorvastatin (Lipitor), fluoxetin (Prozac), and sildenafil (Viagra). The information was compiled from multiple sources after an extensive literature search. For example, the search revealed that ingested acetylsalicylic acid (ASA) is converted to salicylic acid (SA) within the stomach and the circulation system. ${ }^{18} \mathrm{SA}$ is then either renally excreted or first converted to one of four known derivatives (salicyl acyl glucuronide, gentisic acid, salicyl phenolic glucuronide, or salicyluric acid) before subsequent renal excretion. ${ }^{19,20}$ The rates of absorption of ASA and SA from the stomach, the rates of conversion from one compound to another, and the rates of excretion of the various ASA derivatives are shown in Figure 4, which were all identified from the relevant literature. $^{18,21-23}$

The constants and modeling formulas, such as those indicated for ASA, were used to automatically produce the concentration data for the compounds in various compartments over time, resulting in a time series for each compound and each compartment affected. Thus the ADME data for a drug takes the form of a two-dimensional matrix of concentrations (eg, in milligrams), where each row corresponds to a distinct time instant and each column represents a compartment in which the concentrations were measured.

\section{Methods}

The integration of pharmacokinetic data with anatomical data is accomplished by applying the integration framework shown in Figure 1. The two core tasks of the framework that need to be implemented in a data-dependent way are the semantic mapping and the visual mapping. The workings of these two components are illustrated in Figure 5.

The anatomical information contained in the ADME data of a drug is given in terms of compartments in which the metabolite measurements were taken, and therefore is different from Terminologia Anatomica (TA), on which the organization of the human body atlas is based. These terms can be retrieved from the header of the ADME data file and fed to a compartment-to-organ mapper that maps each compartment to corresponding standardized organ names and TA identifiers. For example, the 'Blood' compartment will be mapped to the organ 'A12.0.00.000' (cardiovascular system) in TA, which determines that the chemical compounds located in the blood will be shown on the cardiovascular system of the atlas. The use of ontological representation was not needed for this mapping, as the number of terms involved did not warrant the added complexity. However, as the number of compartments and the number of corresponding organs grow, it will eventually be necessary to adopt ontologies.

The visual mapper needs to produce visual features for expressing different chemical compounds and their concentrations contained in the ADME data. We chose to use colors and their intensities to represent the compounds and their concentrations. First, unique base colors (eg, red, green, or blue) are assigned to each chemical compound (or a small group of a few compounds if their behaviors are similar) appearing in the data. Then the color of an organ at a given 


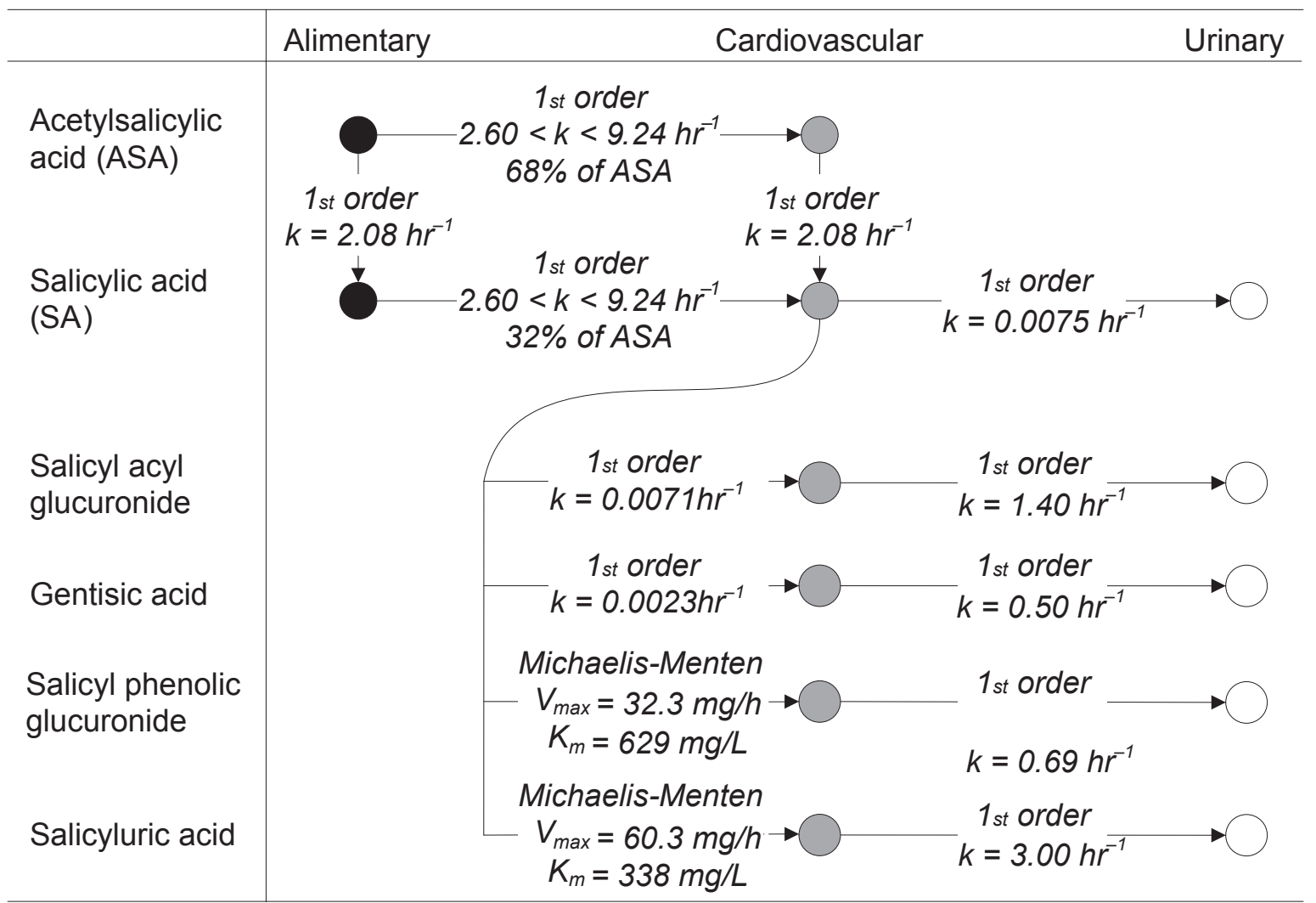

Figure 4 The ADME flow of acetylsalicylic acid in three anatomical systems, corresponding to the initial ingestion, blood concentration, and urine concentration. Either the first order elimination or the Michaelis-Menten kinetics with the specified parameters is used for modeling changes over time in chemical compounds themselves, in concentration, and in affected anatomical systems.

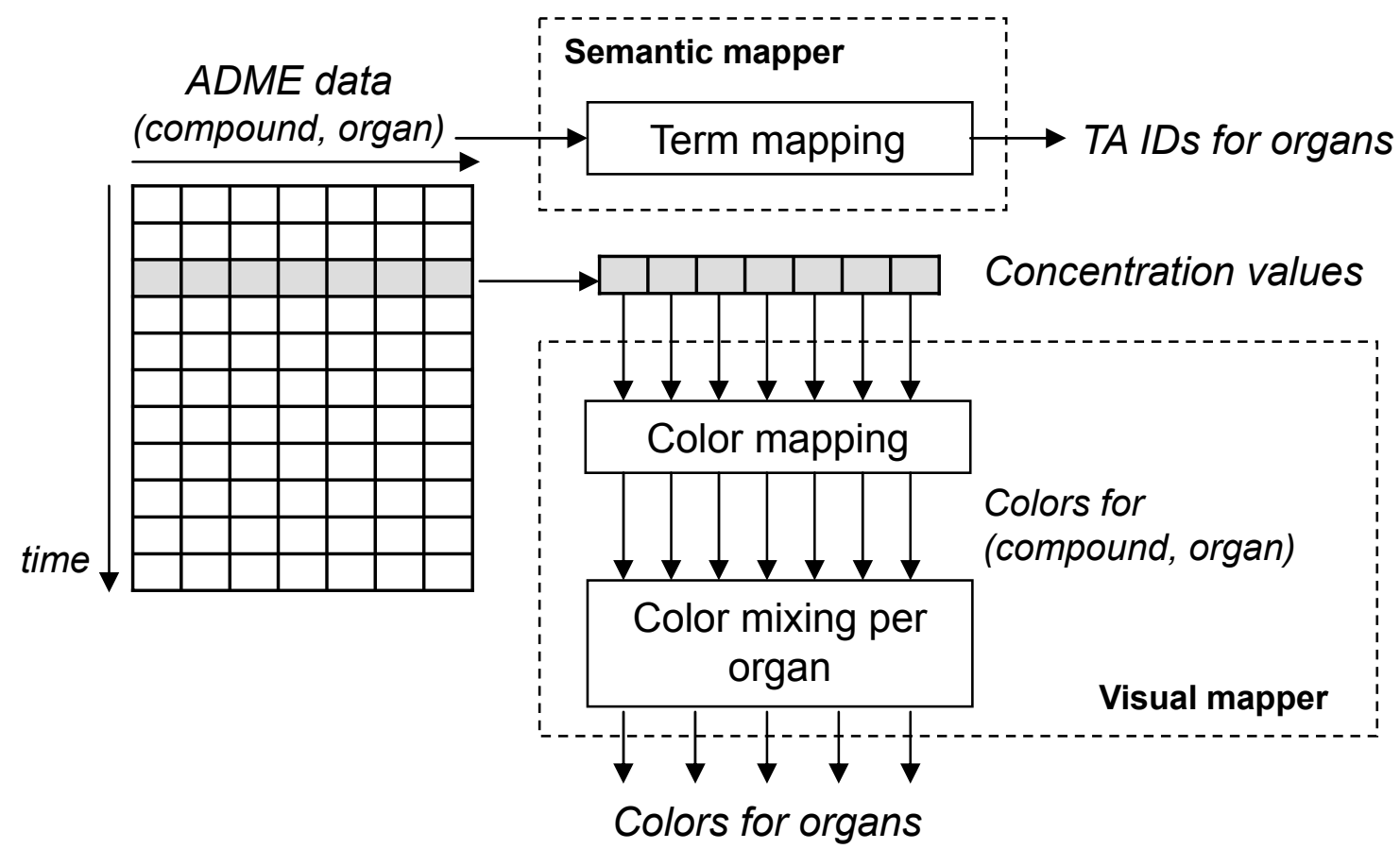

Figure 5 Semantic mapping and visual mapping for integrated visualization of pharmacokinetic data for a single time point. The ADME data is given as a matrix where each cell represents the numerical concentration value of a chemical compound existing in an organ at a time instant. The semantic mapper maps compartments to organ IDs in TA. The visual mapper converts each concentration value to a color value and combines them into a mixed color for each organ. 
time is calculated by scaling the base color of the compound existing in that organ at that time instant by its relative concentration, such that darker colors stand for heavier concentrations. If multiple compounds exist simultaneously in an organ at a certain time point, the colors depicting the different compounds are mixed to indicate the mixture of compounds. This process effectively converts a numerical concentration value of a compound into a unique color value representing the compound and its concentration as well.

\section{Results}

The semantic mapper and visual mapper illustrated in Figure 5 accomplish the core part of the pharmacokinetic data mapping. The mapping results are visualized within the virtual reality environment offered by the CAVEman system. After the desired anatomical systems are loaded into the visualization scene, the user loads the text file that contains the ADME data of a particular drug, which triggers the series of mapping processes required for visual integration of the data. Figure 6 illustrates the interface for the loading of acetaminophen (Tylenol) ADME data into the visualization system. This particular data has 11 (compound, organ) pairs after compartment-to-organ mapping was performed, over 1001 discrete time instants, resulting in a total of 11,011 concentrations to visualize. Currently loaded anatomical models are displayed in the left panel according to the TA hierarchy, with those affected by the drug shown in red. By selecting an anatomical organ or system, the user can see the list of the chemical compounds that exist in the model at some time points on the right. The bottom panel controls the visualization parameters such as the base colors to assign to compounds and the time steps for playing the animation of the ADME process.

The spatiotemporal visualization shows the dynamically changing mixture of colors in the selected body compartments to illustrate the ADME process. Figure 7 shows three typical snapshots of the ADME visualization using 3D models of the

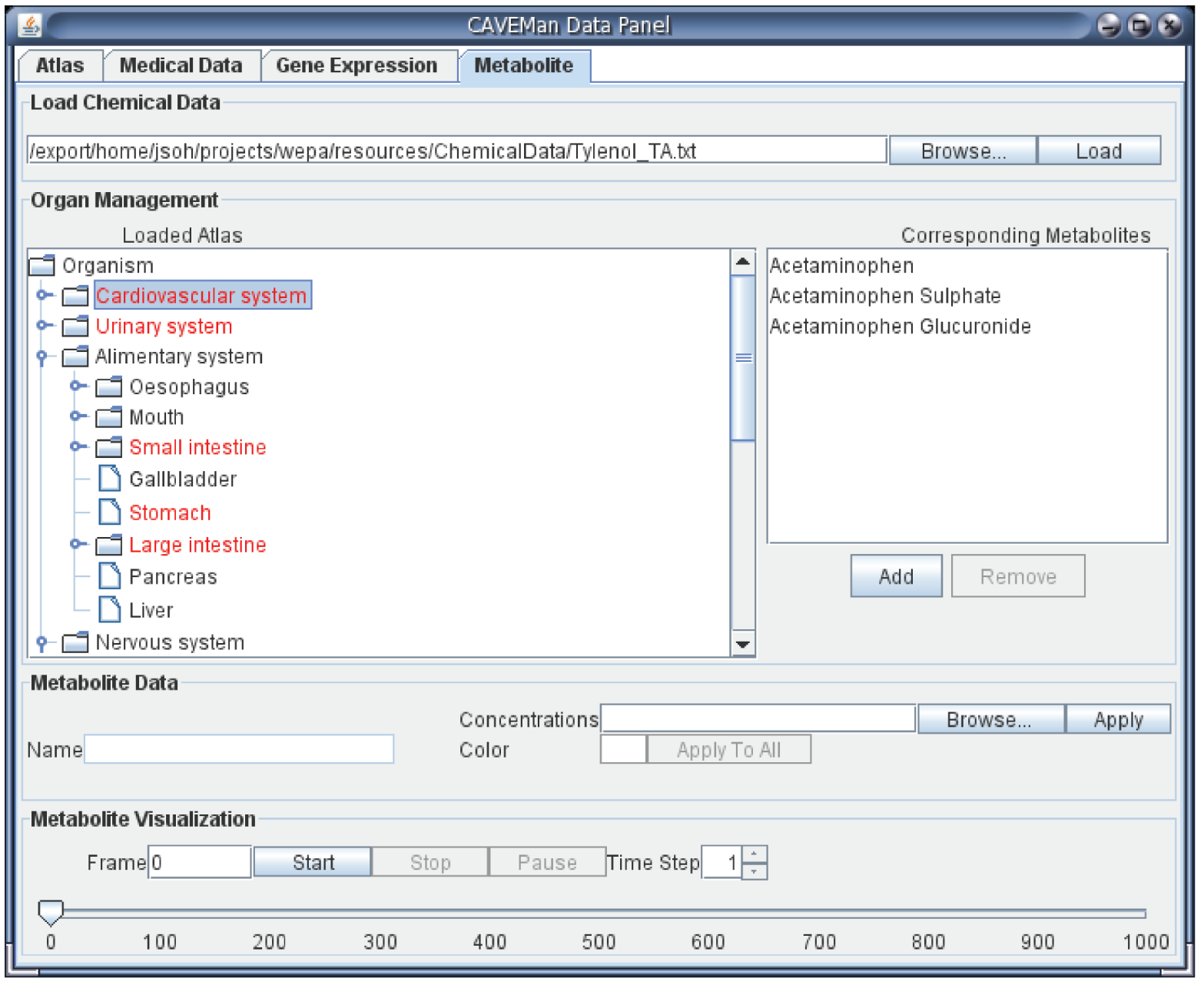

Figure 6 Graphical user interface for the visualization of the acetaminophen ADME process. 


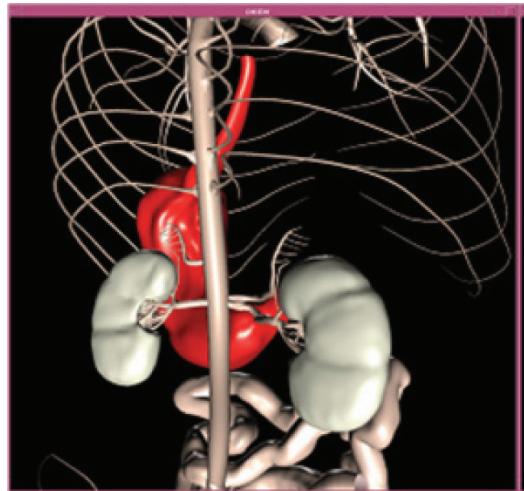

a)

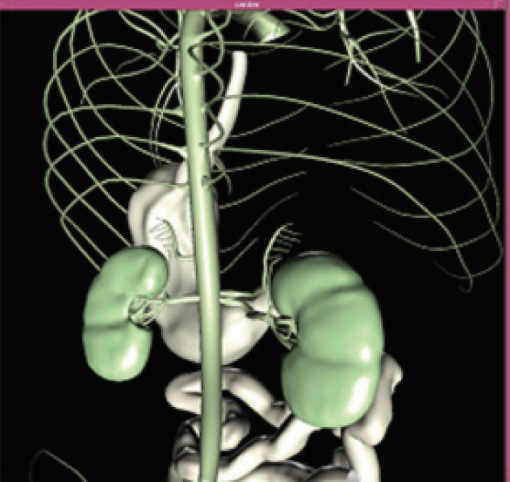

b)

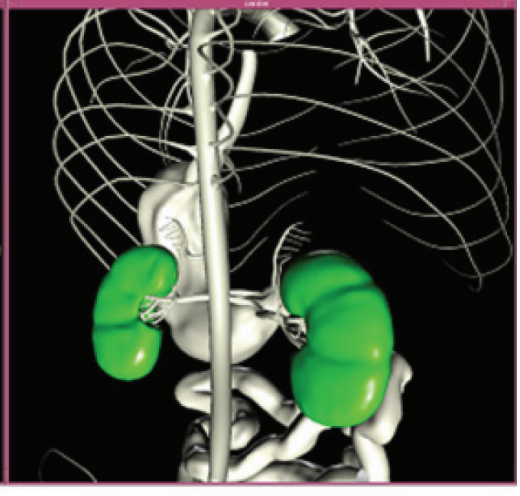

c)

Figure 7 Integrated visualization of pharmacokinetics data on 3D human body. Different metabolites are displayed with different shades of color corresponding to concentrations: a) initial absorption into the digestive system; b) distribution and metabolism throughout the cardiovascular system; and (c) the elimination and excretion of by-products through the urinary system.

stomach, small intestine, arterial structures and kidneys. The original drug compound (red) is shown to be first absorbed through the digestive organs. Simultaneously with its absorption into the blood stream, it is metabolized into derivative compounds (green), which are distributed throughout the body. Eventually all compounds are eliminated from the blood and are excreted through the urinary system. The mixture of red and green color components for each organ is dynamically updated to animate the time series values in the ADME data. This way of visual representation eliminates the need for the user to mentally keep track of multiple concentrations existing in multiple organs over a period of time, which essentially forms a three-dimensional matrix of concentration values, to make sense of the ADME data.

Our integration framework is successful in producing the pharmacokinetic data visualization results to guide researchers through a complex set of numerical data, and allow scientists of various backgrounds to share insights, which normally could only be grasped by a few specialists. The anatomical context is quite important as a communication vehicle within the medical community, as it can be presumed to be part of the background knowledge of everyone involved in the respective study.

\section{Discussion}

Our framework is unique in that it combines semantic mapping and visual mapping into an integrated visualization of the biomedical data at hand. There were studies on biomedical data integration that mostly focused on lexical and semantic mapping of data elements contained in a biomedical database to another database by combining schema-based and instance-based approaches ${ }^{7}$ or using an ontology processing tool. ${ }^{24}$ While our case studies utilize only two independent data sources at a given time (ie, anatomical plus genomic datasets or anatomical plus metabolomic datasets), the integration framework is generic enough to accommodate a larger number of data sources, as long as they are semantically related and can be mapped directly or represented by interrelated ontologies. Examples of such data sources are multiple gene expression datasets, metabolomic datasets, and medical imaging datasets.

To our knowledge, comparable work on visually integrating anatomical data and other types of biomedical data is scarce. BioMediator ${ }^{25}$ is a data integration system that has been applied to link four heterogeneous neurophysical data sources for the needs of neuroscience researchers. Although it uses the Foundational Model of Anatomy (FMA) ontology, ${ }^{26}$ there is no visual component that puts the integrated data in an anatomical perspective. FMA has also been used as the central anatomical ontology for integrating the Gene Ontology (GO; http://www.geneontology.org) and the Mouse Genome Informatics (http://www.informatics.jax.org). ${ }^{27}$ This work is similar to ours in that it used anatomy as a hub for combining different genomic knowledge sources, but FMA only functions as an information portal for browsing and querying. For example, there is no visualization of gene expression values in the anatomical context.

Our work demonstrates that converting biomedical data into ontologies, as we have done for the SymAtlas gene expression dataset, provides a number of technological benefits. The process of transforming ontologies into OWL has recently been described from the biological and technical perspective. ${ }^{28}$ An important advantage of OWL encoding is that new relationships can be automatically inferred using first-order logic, which is key to minimizing the manual effort required in defining relationships between data sources. In 
contrast, traditional data integration efforts would create either a global schema to which all data providers map their data or field mappings between each pair of databases. The former diminishes data-provider independence and limits the view of the underlying data, whereas the latter introduces combinatorial complexity and requires strict lexical identity among the data sources to enable queries. Our ontology-based semantic mapping facilitates modular architecture in which data sources can be completely independent.

The semantic mapping aspect of the integration framework deals mostly with anatomical information, whereas the visual mapping module converts the remaining symbolic information into a visually meaningful form. The choice of visual features is of critical importance, as the features should have sufficient dimensionalities to be able to represent the variability in the data values present in the symbolic information, and yet they should be easily rendered in the visualization environment. We chose color as the main visual feature in both case studies because it has at least two visually (as opposed to colorimetrically) distinct dimensions: the hue and shade. For example, this choice has helped us to successfully render all possible mixtures of multiple chemical compound concentrations by using a hue to represent a compound itself and different shades to represent concentrations.

The importance of knowledge integration has been widely understood in the narrower area of bioinformatics, which focuses predominantly on molecular studies. ${ }^{29,30}$ The broader field of nanomedicine presents an even more challenging area for data integration: indeed, complex biomedical phenomena involve diverse and seemingly unrelated data from several domains of knowledge, each of which has already spawned a variety of data formats and vastly different scales, from a nanoscale to a macroscale. The emphasis on visualization as a data integration platform leads to natural development of a practical data integration framework where the nature of semantic mapping as well as visual mapping is defined by the characteristics of the biomedical data sources to be integrated.

The importance of our framework lies in presenting a methodological template for the visual spatiotemporal modeling of molecular events in their proper anatomical context. The two case studies demonstrate that this framework, based on mapping of data semantics and judicious use of visual features, enables spatiotemporal visualization of complex biomedical phenomena. 3D graphical representation provides users with high-level models that are self-explanatory and lead to further investigation into the raw data. Visualization of biomedical data in a proper context can help researchers to obtain a comprehensive view of the massive complexity of multiple incompatible data types and focus instead on developing a highly intuitive understanding of the studied phenomena. Information visualization has already become one of the main techniques in bioinformatics, and we anticipate that its importance will only increase.

\section{Conclusions}

We have described a systematic framework for bioinformatics data integration based on integration of semantic mapping and visual mapping results. We use ontologies to semantically (as opposed to lexically) resolve the ambiguities stemming from different sets of uncontrolled terminologies. Semantic mapping is accomplished by either using a simple term mapper or an ontology reasoning engine to process semantic relationships contained in the ontologies. Visual mapping transforms the textual or numerical data into visually distinct and intuitive representations that can readily be shown in our virtual reality environment. The integrated framework provides users with an effective way to exploit, analyze, and interact with their data, as well as to potentially embed other data analysis tools.

This work is part of the existing CAVEman Human Body project, which uses immersive virtual reality visualization and animation to represent biomedical data of varying granularity, including spatiotemporal processes. The 3D data visualization component of the integration framework allows researchers to load, view, and interact with data objects ranges from molecular complexes to $3 \mathrm{D}$ atlases of different organisms. As a result of semantic and visual data integration, users can take advantage of the CAVEman capabilities to explore the related biological data from different data sources across different organisms in an integrated visual environment and perhaps generate new biological insights.

\section{Acknowledgments}

This work has been supported by Genome Canada through Genome Alberta; the National Research Council of Canada's Industrial Research Assistance Program; the Alberta Science and Research Authority; Western Economic Diversification; the Government of Canada and the Government of Alberta through the Western Economic Partnership Agreement; the iCORE/Sun Microsystems Industrial Research Chair program; the Alberta Network for Proteomics Innovation; and the Canada Foundation for Innovation. J. Xia is supported by an Ingenuity $\mathrm{PhD}$ Student Scholarship from the Alberta Ingenuity Fund. 


\section{References}

1. Turinsky AL, Sensen CW. On the way to building an integrated computational environment for the study of developmental patterns and genetic diseases. Int J Nanomedicine. 2006;1:89-96.

2. Batini C, Lenzerini M, Navathe SB. A comparative analysis of methodologies for database schema integration. ACM Comput Surv. 1986;18:323-364.

3. Turinsky AL, Fanea E, Trinh Q, et al. CAVEman: standardized anatomical context for biomedical data mapping. Anat Sci Ed. 2008;1:10-18.

4. Lajeunesse D, Edwards C, Grosenick B. Realism: a study in human structural anatomy. Red Deer: Kasterstener Publications Inc; 2003.

5. Federative Committee on Anatomical Technology. Terminologia Anatomica - international anatomical terminology. Stuttgart, New York: Thieme; 1998.

6. Whitmore I. Terminologia Anatomica: new terminology for the new anatomist. Anat Rec. 1999;257:50-53.

7. Mougin F, Burgun A, Bodenreider O. Mapping data elements to terminological resources for integrating biomedical data sources. BMC Bioinformatics. 2006; 7:S6.

8. Barrett T, Troup DB, Wilhite SE, et al. NCBI GEO: Mining tens of millions of expression profiles - database and tools update. Nucleic Acids Res. 2007;35:D760-D765.

9. Su AI, Wiltshire T, Batalov S, et al. A gene atlas of the mouse and human protein-encoding transcriptomes. Proc Natl Acad Sci USA. 2004;101:6062-6067.

10. Wu Z, Irizarry RA, Gentleman R, et al. A model-based background adjustment for oligonucleotide expression arrays. J Am Stat Assoc. 2004;99:909-917.

11. Lockhart DJ, Dong H, Byrne MC, et al. Expression monitoring by hybridization to high-density oligonucleotide arrays. Nat Biotechnol. 1996;14:1675-1680.

12. Gruber TR. A translation approach to portable ontology specifications. Knowl Acquis. 1993;5:199-220.

13. Bodenreider O, Stevens R. Bio-ontologies: Current trends and future directions. Brief Bioinform. 2006;7:256-274.

14. Sirin E, Parsia B, Grau BC, et al. Pellet: A practical OWL-DL reasoner. $J$ Web Semantics. 2007;5:51-53.

15. Wishart DS, Tzur D, Knox C, et al. HMDB: the Human Metabolome Database. Nucleic Acids Res. 2007;35:D521-D526.
16. Wishart DS, Knox C, Guo AC, et al. DrugBank: a knowledgebase for drugs, drug actions and drug targets. Nucleic Acids Res. 2008;36: D901-D906.

17. Gibaldi M, Perrier D, editors. Pharmacokinetics. New York: Informa Healthcare; 1982.

18. Rowland M, Riegelman S, Harris PA, et al. Absorption kinetics of aspirin in man following oral administration of an aqueous solution. J Pharm Sci. 1972;16:379-385.

19. Tsuchiya T, Levy G. Biotransformation of salicylic acid and its acyl and phenolic glucuronides in man. J Pharm Sci. 1972;61:800-801.

20. Liu J, Smith PC. Direct analysis of salicylic acid, salicyl acyl glucuronide, salicyluric acid and gentisic acid in human plasma and urine by high-performance liquid chromatography. J Chromatogr. 1996;675:61-70.

21. Levy G, Tsuchiya T, Amsel LP. Limited capacity for salicyl phenolic glucuronide formation and its effect on the kinetics of salicylate elimination in man. Clin Pharmacol Ther. 1972;13:258-268.

22. Bochner F, Graham GG, Cham BE, et al. Salicylate metabolite kinetics after several salicylates. Clin Pharmacol Ther. 1981;30:266-275.

23. Needs CJ, Brooks PM. Clinical pharmacokinetics of the salicylates. Clin Pharmacokinet. 1985;10:164-177.

24. Narayanan PS, O'Connor MJ, Das AK. Ontology-driven mapping of temporal data in biomedical databases. AMIA Annu Symp Proc. 2006; 1045 .

25. Wang K, Tarczy-Hornoch P, Shaker R, Mork P, Brinkley JF. BioMediator data integration: beyond genomics to neuroscience data. AMIA Annu Symp Proc. 2005;789-783.

26. Rosse C, Mejino JVL. A reference ontology for biomedical informatics: the Foundational Model of Anatomy. J Biomed Inform. 2003;36:478-500.

27. Gennari JH, Silberfein A, Wiley JC. Integrating knowledge sources through and anatomy ontology. In: Pacific Symposium on Biocomputing; 2005 Jan 4-8; Hawaii, USA. p. 115-126.

28. Aranguren $\mathrm{M}$, Bechhofer $\mathrm{S}$, Lord $\mathrm{P}$, et al. Understanding and using the meaning of statements in a bio-ontology: recasting the gene ontology in OWL. BMC Bioinformatics. 2007;8:57.

29. Chicurel M. Bioinformatics: bringing it all together. Nature. 2002;419:751,753-755.

30. Stein L. Creating a bioinformatics nation. Nature. 2002;417:119-120. 
